\title{
INVESTIGACIONES
}

\section{Abandono de la profesión docente en Chile: Motivos para irse del aula y condiciones para volver*}

\author{
Teacher attrition in Chile: \\ Reasons for leaving the classroom and conditions for coming back
}

\author{
Alfredo Gaete Silva, ${ }^{a}$ María Castro Navarrete ${ }^{a b}$ \\ Felipe Pino Conejeros, ${ }^{a c}$ Diego Mansilla Devia ${ }^{a d}$ \\ aPontificia Universidad Católica de Chile, Campus Villarrica \\ Telf.: (56) 452411830. Correo electrónico: agaetes@uc.cl
}

'Telf.: (56) 452411830. Correo electrónico: lorecastronavarrete@ gmail.com

cTelf.: (56) 452411830. Correo electrónico: felipe.pino.conejeros@gmail.com

dTelf.: (56) 973313813. Correo electrónico: damansil@uc.cl

\begin{abstract}
Chile está dentro de los países que registran las tasas más altas de abandono docente. Sin embargo, la investigación en esta materia ha sido escasa hasta ahora. En particular, poco sabemos sobre las razones por las cuales nuestros profesores deciden dejar el aula. En este artículo se presentan los resultados de un estudio cualitativo cuyo propósito fue ayudar a la comprensión de este fenómeno. A partir de entrevistas realizadas a 28 profesionales de la enseñanza básica que dejaron de hacer clases para dedicarse a otras actividades, se encontró que las motivaciones más frecuentes para tomar esa decisión son tener otros intereses, asumir un cargo de gestión, la insatisfacción con las remuneraciones y las condiciones laborales de la escuela, la sensación de no ser considerado como profesional y la prosecución de estudios de perfeccionamiento. También hay desmotivación producida por el influjo que ha tenido la medición estandarizada sobre el sistema escolar.
\end{abstract}

RESUMEN

Palabras clave: abandono docente, desarrollo profesional docente, educación chilena.

\section{ABSTRACT}

Chile is among the countries with the highest teacher attrition rate. However, at present there is not much research on this issue. In particular, we know little about the reasons why our teachers decide to leave the classroom. In this paper we present the results of a qualitative study, the purpose of which was to contribute to the understanding of this phenomenon. By means of 28 interviews conducted with primary teachers who had quitted teaching, we found that the most frequent motivations for making that decision are having other interests, taking a management position, dissatisfaction with wages and working conditions at school, a sensation of not being considered as a professional, and the pursuit of further studies. There is also a disenchantment stemming from the influence of standardized measurements upon the school system.

Key words: teacher attrition, teacher professional development, Chilean education. 


\section{INTRODUCCIÓN}

En muchos países el abandono de la profesión docente ha registrado un aumento bastante preocupante, especialmente durante los primeros años de ejercicio (Ávalos \& Valenzuela, 2016; Correa et al., 2013; Ingersoll, 2002; Ingersoll \& Strong, 2011; Manuel, 2003; MacBeath, 2012; OECD, 2001; Zehm, 1999). Dentro de las razones que motivan a los profesores a dejar el aula, las más mencionadas en la literatura internacional son las siguientes:

(a) Percepción, por parte de los profesores en servicio, de una desprofesionalización de la labor docente, asociada a una sensación de falta de confianza en ellos en tanto profesionales de la enseñanza y a una escasa autonomía para la toma de decisiones; por ejemplo, en la imposición de actividades, contenidos o programas (MacBeath, 2012; PricewaterhouseCoopers, 2001).

(b) Insatisfacción laboral de los profesores, generalmente asociada a un mal ambiente de trabajo con recursos y apoyo insuficientes (Centro de Políticas Públicas, 2012; Klassen \& Chiu, 2010; MacBeath, 2012; PricewaterhouseCoopers, 2001; Struyven \& Vanthournout, 2014). Esto se conecta con el "choque de realidad" (Veenman, 1984) que sufren algunos docentes principiantes durante sus primeras experiencias laborales (véase también Ávalos, 2013). En general, parece haber una asociación bastante clara entre inserción profesional difícil y abandono (Correa et al., 2013; Darling-Hammond, 2003; Lortie, 2002; Manuel, 2003; Taylor \& Dale, 1971).

(c) Sobrecarga laboral de los profesores (Barmby, 2006; MacBeath, 2012; McKenzie, Santiago, Sliwka \& Hiroyuki, 2005; PricewaterhouseCoopers, 2001; Veenman, 1984), que puede generar o contribuir a generar casos de burnout (Grossman \& Thompson, 2004; Skaalvick \& Skaalvick, 2011).

(d) Dificultades asociadas al mal comportamiento de los estudiantes (Barmby, 2006; MacBeath, 2012; McKenzie et al., 2005).

(e) Dificultades asociadas a la diversidad de los estudiantes (Hancock \& Scherff, 2010; MacBeath, 2012).

(f) Temas salariales (Hancock \& Scherff, 2010; Hanushek, Kain \& Rivkin, 2004; Luekens, Lyter \& Fox, 2004).

Chile parece estar dentro del grupo de países con mayores dificultades para retener a sus docentes, registrando una tasa de abandono de alrededor del $40 \%$ durante los primeros 5 años de ejercicio profesional (Ávalos \& Valenzuela, 2016; Valenzuela \& Sevilla, 2013). Sin embargo, la información con que contamos sobre las motivaciones de estos profesionales para dejar el aula es escasa. No sabemos, por ejemplo, hasta qué punto su decisión se debe a las razones antes mencionadas ni qué otras razones puedan tener. Mizala et al. (2000) encontraron que el "grado de satisfacción con la carrera" era la variable más influyente, pero no queda claro a qué se refieren exactamente con eso: ¿a las malas condiciones de trabajo, a la falta de proyección profesional, a asuntos vocacionales, a una combinación de estas y quizá otras razones? Otra motivación relevante podría ser la inconformidad con el salario, pero tampoco hay claridad sobre esto, no solo porque la relación estadística que establecería este hallazgo es significativa únicamente al 10\%, sino también porque los participantes de este estudio eran profesores en servicio a quienes se preguntó si habían pensado alguna vez en dejar la profesión, no profesores que la habían dejado de hecho. Esta 
última consideración se aplica también a lo encontrado respecto del grado de satisfacción con la carrera.

Un estudio reciente de Ávalos y Valenzuela (2016) ha sido un avance en este sentido. Los participantes del estudio son docentes que efectivamente habían dejado de hacer clases, a quienes se aplicó un cuestionario que pedía especificar la importancia de distintas posibles razones para abandonar la profesión. En virtud del número de profesores que escogían las alternativas "muy importante" y "extremadamente importante", los investigadores identificaron tres áreas especialmente relevantes: oportunidades de desarrollo profesional, condiciones laborales y gestión de la escuela. Las 10 razones que más fueron consignadas, en orden decreciente, son

(a) Insatisfacción con las oportunidades de desarrollo profesional disponibles.

(b) Ingreso insuficiente para satisfacer las necesidades vitales.

(c) Falta de beneficios complementarios adicionales adecuados.

(d) Insatisfacción con el liderazgo de la gestión.

(e) Insatisfacción con las condiciones de la escuela (seguridad, materiales, etc.).

(f) Falta de influencia sobre las políticas y prácticas de la escuela.

(g) Deseo de mejorar el estándar de vida.

(h) Insuficiente libertad para tomar decisiones pedagógicas al hacer clases.

(i) Deseo de seguir estudiando para tener más oportunidades en el campo educacional.

(j) Número de estudiantes demasiado alto.

En este artículo presentamos los resultados de un estudio cuyo propósito fue, precisamente, comprender con mayor profundidad estas y otras motivaciones que llevan a los profesores de enseñanza básica del sistema educativo chileno a tomar la decisión de dejar el aula. ¿En qué sentido nuestras escuelas no están entregando a los profesores la oportunidad de desarrollarse profesionalmente? ¿Qué es exactamente lo que no les gusta de la gestión de los directivos? ¿Es el ingreso un factor en sí mismo o se vuelve tal en virtud de las malas condiciones de trabajo? ¿Qué otras razones no detectadas aún podrían estar operando?

En la literatura sobre abandono docente suele distinguirse entre los profesores que abandonan de manera voluntaria y aquellos que se ven más bien obligados a hacerlo, como ocurre en el caso de la jubilación o de la maternidad, por ejemplo (Struyven \& Vanthournout, 2014). Dentro del primer grupo se distingue también entre quienes deciden migrar a otro establecimiento y quienes dejan la profesión docente (Billingsley, 1993). Poco o nada se dice, sin embargo, de quienes dejan el aula pero no la escuela (usualmente para tomar algún cargo de gestión) o de quienes dejan el aula y la escuela pero no la educación (para ocupar, por ejemplo, alguna posición en el Ministerio de Educación o en fundaciones educacionales o en instituciones formadoras de profesores, entre otras posibilidades). En adelante nos referiremos a estos dos tipos de abandono como "tipo 1" y "tipo 2", respectivamente; y hablaremos de "tipo 3" para aludir a quienes deciden dejar no solo el aula y la escuela, sino también la educación. El estudio que aquí reportamos está restringido a estos 3 tipos de abandono a nivel de la educación primaria en Chile, esto es, a profesores de enseñanza básica que han abandonado voluntariamente su labor como tales. Por qué creemos que es importante distinguir estos 3 tipos de abandono será parte de la discusión. 


\section{MÉTODO}

Participaron en el estudio 28 profesores de básica que habían dejado de ejercer su profesión en establecimientos educativos ubicados en distintas regiones del país (Coquimbo, Metropolitana, Biobío, La Araucanía y Los Lagos). De ellos, 10 tuvieron oportunidad de trabajar en colegios particulares pagados, 18 en colegios subvencionados y 13 en escuelas municipales. Además, 22 se desempeñaron en sectores urbanos, 11 en sectores rurales y solo 5 en ambos sectores. El número de años de ejercicio fluctuó entre 1 y 29, pero la mayoría de los participantes se retiró antes de los 10. Se incluyeron casos de abandono de tipo 1,2 y 3.

Desde un enfoque metodológico cualitativo, se llevaron a cabo entrevistas semiestructuradas con cada uno de los participantes. En todas ellas se indagó al menos en 3 áreas. Primero, en las razones por las cuales los participantes decidieron dejar el aula. Para ello, el entrevistador preguntaba directamente: “¿Cuáles fueron sus razones para dejar de hacer clases en básica?” Luego pedía a los participantes que compararan su ocupación actual con su trabajo como profesores de básica: “¿Qué le gusta de su trabajo/ocupación actual que no tenía cuando hacía clases en la escuela?" Finalmente, interesaba explorar las condiciones bajo las cuales los participantes estarían dispuestos a retomar la labor docente en el sistema escolar, para lo cual el entrevistador preguntaba: “¿Volvería a hacer clases en básica? ¿Bajo qué condiciones?”

Las entrevistas fueron grabadas y posteriormente analizadas por un equipo compuesto siempre por al menos 3 investigadores (a veces 4), quienes categorizaron las respuestas de los participantes. Todos los investigadores tenían experiencia en educación. Tanto para generar las categorías como para ubicar cada una de las respuestas en ellas, se consideró necesario que hubiese un acuerdo unánime entre los investigadores. Esto llevó a que el proceso de categorización se extendiera durante varios meses, en sesiones de discusión que duraban entre 1 y 3 horas.

\section{RESULTADOS}

A continuación presentamos los resultados obtenidos en las tres áreas en las que se indagó en las entrevistas. Primero presentamos una tabla que muestra las categorías que fueron más aludidas por los participantes, para luego explicar cada una de estas categorías y ofrecer algunos extractos de las entrevistas que ayudan a comprenderlas mejor. Los extractos se presentan entrecomillados, seguidos por un paréntesis que especifica el género del participante, el número de años durante los cuales hizo clases en básica y el número de años que lleva fuera del aula al momento de la entrevista. Los puntos suspensivos entre corchetes indican que se omitieron partes del reporte (usualmente en pro de la claridad). También entre corchetes se registran las intervenciones de los entrevistadores y algunos agregados que a juicio de los investigadores permiten entender mejor las ideas expresadas.

\subsection{RAZONES PARA DEJAR EL AULA}

La Tabla 1 muestra las categorías que más aparecieron en respuesta a la pregunta sobre las razones para abandonar la enseñanza. 
Tabla 1. ¿Cuáles fueron sus razones para dejar de hacer clases en básica?

\begin{tabular}{|l|c|}
\hline \multicolumn{1}{|c|}{ Categoría } & $\begin{array}{c}\text { Número de participantes que } \\
\text { hicieron alusión a ella }\end{array}$ \\
\hline Otros intereses & 13 \\
\hline Asumió un cargo de gestión & 8 \\
\hline Remuneraciones & 7 \\
\hline Insatisfacción laboral & 6 \\
\hline Sobrecarga de trabajo & 5 \\
\hline Desprofesionalización & 4 \\
\hline Perfeccionamiento & 4 \\
\hline
\end{tabular}

\subsubsection{Otros intereses}

En esta categoría se incluyeron referencias que los entrevistados hacían a intereses distintos de enseñar en la escuela: "Yo sentía la necesidad de crear más allá de la clase propiamente tal... A mí me gustaba — me gusta- mucho la gestión: me gusta inventar proyectos, más allá del aula" (mujer, 14 años dentro, 1 año fuera). En algunos casos, el cambio de rumbo ocurría de manera paulatina: "Luego de que salí [de la universidad] trabajé un tiempo, comencé con el magíster y [...] distintas actividades que eran ajenas a la pedagogía propiamente tal me fueron llevando por otro camino [...] Fue algo que se fue dando [...] por mi afición a hacer otro tipo de actividades y proyectos" (hombre, 1 año dentro, 3 años fuera). Había incluso quienes parecían nunca haber estado muy interesados en el trabajo de aula: "En realidad, siempre mi interés, desde haber salido de la universidad, fue el tema de la investigación, más que todo" (hombre, 4 años dentro, 15 años fuera). Otra profesora señala: "Yo no sé si mi vocación era la pedagogía [...] Me encanta, pero también alucino con lo que estoy haciendo [...] No estoy frustrada. Estoy de hecho bien contenta, porque a mí me cuesta mucho estar encerrada: esta cuestión de estar en el colegio [...] y de responder a horarios [...] y la directora... Prefiero no estar ahí" (mujer, 14 años dentro, 1 año fuera). La falta de vocación también fue referida en otro relato: "Esa cosa que llaman vocación yo no la reconozco. A mí no me la pusieron al nacer [...] Yo soy el prototipo de la persona que [...] no le dio para ser abogado y se metió a estudiar [pedagogía]" (mujer, 10 años dentro, 15 años fuera).

\subsubsection{Asumió un cargo de gestión}

Esta categoría aúna las respuestas que apuntaban al hecho de haber ocupado algún cargo de gestión en un colegio: dirección, jefatura de U.T.P., inspectoría, etc. Si bien estos cargos no son necesariamente incompatibles con la docencia, en los casos aquí consignados se apelaba a ellos como razón para dejarla, y en la mitad ellos como la única razón: "La razón fue que me ofrecieron el cargo de jefa técnica. Esa fue la única razón, porque hacer clases me encanta" (mujer, 8 años dentro, 3 años fuera). Naturalmente, esta razón fue referida solo en casos de abandono de tipo 1 . 


\subsubsection{Remuneraciones}

Aquí se consignaron distintas expresiones de insatisfacción por parte de los entrevistados con respecto a la retribución económica recibida por su desempeño como docentes. Cabe señalar que ninguno de los participantes apuntó al tema económico como única razón para abandonar el aula: siempre apareció junto a otra u otras razones y nunca fue la primera de ellas en ser mencionada. Además, algunos participantes matizaban su influencia: "La vida me fue dando otras oportunidades, como trabajar en una ATE [...] El sueldo no sé si fue tan predominante, pero obviamente las mejores ofertas incluían alzas de sueldo [y] creo que eso también me llevó por este camino" (hombre, 2 años dentro, 2 años fuera). Otro entrevistado, después de aclarar que el aspecto económico no fue para él una razón de peso, agrega: "Pero dada la gran cantidad de trabajo que demandan el sistema municipal y el sistema particular subvencionado, versus la remuneración, claro que es mala" (hombre, 2 años dentro, 3 años fuera).

\subsubsection{Insatisfacción laboral}

Los reportes agrupados bajo este concepto aludían a una sensación de insatisfacción con una o más condiciones de trabajo en los colegios: mal clima laboral, falta de recursos, poco apoyo por parte de los encargados de la gestión, excesiva burocracia administrativa, etc. Por ejemplo: "La principal [razón para dejar de hacer clases en básica] es las condiciones: el poco tiempo, las condiciones de vida, el deterioro de la salud, entre otras cosas. [El trabajo] se transformó en llenar papeles y de hacer clases casi nada. Entonces, si seguía en esto era para enseñar, no para llenar papeles" (mujer, 7 años dentro, 1 año fuera). El tema de la salud aparece sugerido también en otro relato: "No me veía haciendo clases todo el tiempo, por el grado de estrés al que los profesores están sometidos, y las condiciones laborales" (mujer, 2 años dentro, 1 año fuera). Otra profesora señala: "No había mucho apoyo de la comunidad escolar para que se hicieran cosas dentro de la sala de clases. [¿Cosas como qué, por ejemplo?] Cosas como coordinar el trabajo con el proyecto de integración, o coordinar el trabajo socio-afectivo en una comunidad con alto índice de vulnerabilidad [...] [¿Qué tipo de apoyo?] Como apoyos [...] desde la escuela... [¿Del equipo directivo?] Claro, del equipo directivo fundamentalmente, pero también en cuanto a tiempos [...] para coordinarse. O sea, yo siempre hice clases no más [...] Me hubiera gustado coordinarme con mis colegas, por ejemplo. [¿Y qué te lo impedía?] Que no teníamos tiempo, que el colegio no nos daba... No había horas comunes de trabajo. [Te hubiera gustado que te hubieran reacomodado el trabajo para...] Que hubiera habido una intención desde la dirección para gestionar también esas problemáticas. [¿Y tú lo hiciste saber?] Sí, lo hice saber. Y no fue acogido. [En un colegio me dijeron] usted es la profesora del colegio, hágase cargo [...] Esa fue una discusión fuerte que tuvimos, porque además era mi primer año de experiencia, entonces yo encontraba súper negligente de parte del [...] sostenedor del colegio que tuviera a alguien de primer año [...] trabajando con niños con problemáticas súper graves" (mujer, 5 años dentro, 5 años fuera).

\subsubsection{Sobrecarga de trabajo}

Esta categoría incluye alusiones al exceso de trabajo y la falta de tiempo libre, que puede tener incluso repercusiones en la vida familiar de los participantes: "El hecho de tener 
familia y que el hacer clases en establecimientos públicos requiere un desgaste fuerte e importante y [...] cuando tú ya tienes hijos no estás dispuesto a llegar en condición de zombi a la casa (hombre, 2 años dentro, 2 años fuera). Otro profesor señala: "Me consumía mucho tiempo. Trabajaba desde las 8 de la mañana hasta las 6 de la tarde, y quedaba lejos [...], así que estaba casi todo el día fuera de la casa”. Buena parte de esta sobrecarga se debe a que los profesores pasan muchas horas de su jornada en el aula, de modo que no les queda tiempo suficiente para planificar clases, preparar materiales, corregir evaluaciones, etc. Esta situación los obliga a hacer horas extraordinarias, muchas veces en casa.

\subsubsection{Desprofesionalización}

Bajo esta rúbrica se ubicaron las respuestas que indicaban como razón para salir del aula una sensación de no ser tratados como profesionales o de tener escasas posibilidades de desarrollo profesional. Esto se asocia al hecho de no poder tomar decisiones de manera autónoma y a no contar con la confianza de los directivos o los sostenedores. Por ejemplo, después de relatar una situación problemática en la que el equipo de gestión del establecimiento no compartió con ella la totalidad de la información relevante disponible, una profesora comenta: "Sentía que no me consideraban un profesional. Porque yo creo que si al profesor tú lo consideras un profesional de la educación, lo mínimo que le puedes dar son las herramientas [como la información disponible]" (mujer, 20 años dentro, 3 años fuera). Otra profesora dice: "Yo creo que mi problema era más como por las instituciones: que eran colegios súper estructurados, en donde tenía que responder a ciertas formas de hacer clases [...] Yo siempre sentía que mi techo estaba muy abajo [...] No podía llegar con toda mi creatividad a dar clases, tenía que hacer clases de una forma determinada [...] Lo que me faltó fue confianza, [que me dijeran] oye, nosotros como colegio confiamos en ti, dale, echa a volar tu imaginación, crea el vínculo con tus alumnos como tú quieras, trabaja con ellos como tú quieras [...]: es tu clase". La figura del "techo bajo" vuelve a aparecer en otro relato: "Encontraba que era poco proyectivo estar dentro de una sala de clases. Encontraba que tenía un techo como muy encima y muy aplastador. Nosotros no reflexionábamos, no pensábamos: cada vez que nos poníamos a discutir [y] a pensar en la sala de profesores, nos interrumpían para ir a hacer clases" (mujer, 10 años dentro, 15 años fuera). La misma profesora agrega más adelante: "Tú vas a hacer clases, sabes lo que tienes que hacer, sabes cómo te lo indican otros, te llevan como en un camino bien ordenadito, con hartas barreras [...] por los lados para que no te vayas a desbocar tratando de ser genial, creativa, ocurrente [...] Yo sentía que uno tocaba el techo sin pegar ni un salto. Y si tú lo miras, yo hoy día [...] veo a mis ex colegas que se han quedado todavía ahí, casi ninguno está haciendo clases. Casi todos aspiraron a otras cosas, que les permitieran mirar el mundo un poco más... No sé, tener más amplitud...”.

\subsubsection{Perfeccionamiento}

En esta categoría se clasificaron los reportes que apuntaban a necesidades u oportunidades de perfeccionamiento que llevaban a los docentes a salir del aula y concentrarse en sus estudios: "Había una beca Conicyt para hacer un magíster en Santiago. Yo trabajaba en Villarrica y me gané la beca, me fui a Santiago y, como la beca tiene un sueldo, dejé de hacer clases, me dediqué solamente a hacer el magíster" (hombre, 3 años dentro, 1 año 
fuera). Otro docente señala: "Siempre quise aspirar a otras opciones profesionales, hacer un magíster y otras cosas, y entre eso encontré esta opción que creo que me acomodaba bastante" (mujer, 2 años dentro, 1 año fuera).

\subsection{COMPARACIÓN CON LA OCUPACIÓN ACTUAL}

La Tabla 2 muestra las categorías que más aparecieron cuando se les pidió a los participantes comparar su trabajo u ocupación actual con su labor docente en aula.

Tabla 2. ¿Qué le gusta de su trabajo/ocupación actual que no tenía cuando hacía clases en la escuela?

\begin{tabular}{|l|c|}
\hline \multicolumn{1}{|c|}{ Categoría } & $\begin{array}{c}\text { Número de participantes que } \\
\text { hicieron alusión a ella }\end{array}$ \\
\hline Desarrollo profesional & 12 \\
\hline Menor carga de trabajo & 12 \\
\hline Impacto & 5 \\
\hline Trabajo en equipo & 4 \\
\hline
\end{tabular}

\subsubsection{Desarrollo profesional}

Esta categoría tiene que ver con poder trabajar en ambientes que ofrezcan oportunidades de desarrollo en las áreas de interés personal, autonomía profesional, libertad de crear e innovar y la posibilidad de aprender: "La disposición de mi lugar de trabajo para que yo pueda desarrollarme profesionalmente [...] en áreas de mi interés. Me interesa que me den permiso para hacer cosas que me permitan seguir profesionalizándome" (mujer, 5 años dentro, 5 años fuera). Otro participante, que actualmente trabaja como docente e investigador en una universidad, señala: "Lo que me gusta de la universidad es que me da la posibilidad de desarrollarme en lo que me gusta, que es [la] investigación, hacer innovación, hacer propuestas, ser más propositivo. En el colegio no se da tanto esa posibilidad" (hombre, 4 años dentro, 15 años fuera). Una profesora que dejó la educación (tipo 3) comenta: "En el colegio el trabajo se torna monótono, siempre era como lo mismo, no te obligaban a perfeccionarte siempre, entonces [...] te quedas estancado. Encuentro que ahora, por el grado de exigencia que tengo, necesito estudiar más, necesito perfeccionarme más, entonces eso me gusta, me gusta estar siempre con metas, con motivaciones importantes" (mujer, 2 años dentro, 1 año fuera).

\subsubsection{Menor carga de trabajo}

Se consignaron aquí diversas alusiones a una jornada laboral que permite tener tiempo libre para descansar o estar con la familia o hacer otras cosas: "En relación a lo que hacía, creo que la carga de trabajo extra es menor. O sea, la pega uno la hace en la oficina, no te llevas 
el trabajo para la casa [...] Hoy día agradezco el hecho de tener más tiempo en mi casa disponible para poder estar $100 \%$ allá" (mujer, 5 años dentro, 2 años fuera). Pareciera ser que lo que más se valora es no tener que hacer horas extra en casa: "Mi trabajo actual es más pesado que el trabajo en aula, pero no te llevas trabajo a la casa" (hombre, 2 años dentro, 3 años fuera). Otro participante señala: "Trabajo medio día: a las 3 y media ya estoy en mi casa, tengo más tiempo para mi familia" (hombre, 1 año dentro, 1 año fuera). Un participante que en la actualidad se desempeña como terapeuta, aprecia el hecho de "simplemente ir a trabajar, terminar mis sesiones [...] sin preocuparme en exceso de lo que tengo que hacer después. [...]. No llevar trabajo para la casa" (hombre, 2 años dentro, 3 años fuera). El mismo entrevistado destaca también la posibilidad de autogestión del tiempo: "Trabajar a mi propio ritmo, manejar mis tiempos, ejercer mi trabajo de manera tranquila y pudiéndome desconectar luego de mi horario de trabajo" (hombre, 2 años dentro, 3 años fuera).

\subsubsection{Impacto}

Estas respuestas apuntan a la posibilidad de hacer cosas que tienen un impacto mayor al que puede obtenerse en el trabajo en aula. Una de las participantes, que dejó el aula para asumir como coordinadora de 23 escuelas rurales en una corporación educacional, comenta: "Hoy día siento que puedo aportar en 23 escuelas [...] Me siento más útil en ese sentido. En cambio, en una sala de clases en un colegio puedo apoyar y aportar a un solo curso acotado" (mujer, 5 años dentro, 2 años fuera). Otra entrevistada, que en la actualidad ocupa un cargo de gestión en una escuela, señala: "Siento que en el lugar que estoy puedo aportar más al aprendizaje de los niños, monitoreando y acompañando a los colegas, pidiendo siempre más, velando por que las cosas se hagan bien" (mujer, 15 años dentro, 12 años fuera). Obviamente, esta categoría no se registró en los casos de abandono de tipo 3. Cabe señalar, sin embargo, que 4 de las 5 respuestas agrupadas en ella provinieron de casos de abandono de tipo 2.

\subsubsection{Trabajo en equipo}

En esta categoría se reúnen alusiones a la oportunidad de formar parte de un trabajo más colaborativo que el que se realiza habitualmente en el aula. Por ejemplo, refiriéndose a su antiguo trabajo como docente, un participante declara: "Lo que no me gustaba era que había poco trabajo en equipo [...] El profe solo se dedicaba [...] a hacer sus clases" (hombre, 3 años dentro, 1 año fuera). Otra participante incluye en su respuesta el hecho de que en su trabajo actual puede interactuar con personas que ella considera altamente calificadas: "La posibilidad de trabajar en equipos colaborativos con una alta formación profesional" (mujer, 5 años dentro, 5 años fuera).

\subsection{CONDICIONES PARA REGRESAR}

La Tabla 3 muestra las categorías que más aparecieron ante la pregunta por las condiciones que tendrían que darse para que los participantes consideraran volver a desempeñarse como profesores de enseñanza básica. 
Tabla 3. ¿Volvería a hacer clases en básica? ¿Bajo qué condiciones?

\begin{tabular}{|l|c|}
\hline \multicolumn{1}{|c|}{ Categoría } & $\begin{array}{c}\text { Número de participantes que } \\
\text { hicieron alusión a ella }\end{array}$ \\
\hline Mejor sueldo & 12 \\
\hline Menos horas lectivas & 10 \\
\hline Proyecto educativo más interesante & 9 \\
\hline Ser tratado como profesional & 8 \\
\hline Menor carga de trabajo & 6 \\
\hline No volvería bajo ninguna condición & 2 \\
\hline
\end{tabular}

\subsubsection{Mejor sueldo}

Si bien la condición más exigida por los participantes es una remuneración económica más apropiada, en ninguno de estos casos se trata de la única condición exigida. En todos los casos se especifica al menos otra condición, y en muchos se entrega una lista de condiciones entre las cuales el aspecto económico es solo uno de los mencionados: "A mí me encantaría hacer clases si yo pudiera tener más horas de preparación de material, si me pagaran más, si los profesores pudiéramos asumir el rol que realmente nos corresponde en cuanto a las decisiones dentro del aula [y] si no existiera el SIMCE" (mujer, 10 años dentro, 24 años fuera). Sin perjuicio de lo anterior, para algunos esta condición es muy importante: "Si me dicen: 'oye, te invitamos a un colegio súper innovador, donde te vamos a confiar plenamente las clases, donde dale full libertad, pero te vamos a pagar la mitad de lo que estás ganando [ahora]', de ningún modo acepto" (mujer, 14 años dentro, 1 año fuera).

\subsubsection{Más horas lectivas}

Estos diez participantes pusieron como condición que se les dieran menos horas en aula y más horas para preparar sus clases, corregir pruebas y hacer otras labores para las cuales normalmente les falta el tiempo: "Debería existir un mayor porcentaje de tiempo para que puedas planificar, realizar guías, revisar pruebas, que no sea solamente clase [...] Que por ejemplo 40 horas no sean 38 dentro de una sala y 2 para consejo de profesores, sino que sea algo más equilibrado" (hombre, 3 años dentro, 1 año fuera). Para algunos esto parece ser incluso más importante que el factor sueldo; por ejemplo, una participante que al momento de la entrevista se desempeña como jefa de UTP declaró que aunque le mantuvieran su sueldo actual (que es mayor al que tenía como profesora de aula) no volvería a hacer clases a menos que alrededor de la mitad de sus horas de contrato fueran horas no lectivas.

\subsubsection{Proyecto educativo más interesante}

En esta categoría se ubicó una serie de requerimientos relacionados con una visión de la educación más profunda o desafiante que la que suele haber en las escuelas. A veces esta demanda tenía que ver con la posibilidad de desmarcarse del SIMCE (como en el caso 
reportado anteriormente, en 3.1.1.) o, relacionado con ello, de la enseñanza "contenidista": "A lo mejor no hacer tanto contenido que se pase a los estudiantes, porque en realidad tienen como poca reflexión [...] No se trata que le enseñemos menos a los estudiantes, sino de que lo que enseñemos lo enseñemos bien. [¿No enfocarse tanto en los contenidos sino en otros aspectos?] Por ejemplo, tener mucho más avanzado el tema de la resolución de problemas, enfocar el currículum hacia ese objetivo. Y es algo complejo porque tiene que ver con la comprensión lectora, entonces ahí debería haber un trabajo en conjunto. Me gustaría que el profesor [tuviera] una autonomía diferente, porque resulta que ahora está el sistema PAC, y eso es clase a clase, todo listo, todo preparado, simplemente vas y reproduces lo que está ahí: eres como un reproductor no más. Entonces no tienes la facilidad de mover un poco tu planificación, no hay libertad de acción, siendo que somos profesionales y estamos preparados para realizar una clase [...] No hay una libertad del profesor para tomar decisiones" (mujer, 2 años dentro, 1 año fuera). Otro participante apunta a un cambio en la cultura escolar como condición para regresar: "Dada la cultura escolar como está formada en Chile — no sé si en otros países será así- se espera que [funcionemos] como máquinas de producción y no como seres humanos educando a otros" (hombre, 2 años dentro, 3 años fuera). Este mismo participante había señalado en un momento previo de la entrevista: "El problema es que mi meta no se condice con la meta que espera el Estado. Mi meta es que el alumno sea feliz y que aprenda, pero a mí lo que me piden es que el alumno se saque notas, y responda a una prueba estandarizada, aunque después de dar la prueba se le olvide todo. A mí el colegio no me pide aprendizaje significativo, me pide resultados para una prueba. Educar no solo para la felicidad sino para la vida. El hecho de que en los colegios los niños vayan solamente a recibir contenidos... Pueden saber toda la materia de matemática, toda la materia de historia, pero a lo mejor no saben relacionarse con otras personas, a lo mejor no saben responder a una situación difícil, no saben transmitir sus conocimientos a la hora de tener hijos, eso no lo aprenden en el colegio; y, a lo mejor por conductas sociales que se restringen tanto por el hecho simplemente de que tienen que recibir contenidos, esos alumnos al final no lo aprenden". En general, las respuestas incluidas en esta categoría contenían implícita o explícitamente una crítica a un sistema escolar en el cual el aprendizaje esperado es superficial o demasiado restringido a ciertos ámbitos, usualmente aquellos que son medidos por las pruebas estandarizadas.

\subsubsection{Ser tratado como profesional}

Se agruparon aquí respuestas que apuntaban a la necesidad de sentir la confianza de los directivos en la capacidad de los profesores para tomar decisiones autónomas en tanto profesionales de la enseñanza: "Que el colegio confiara plenamente en mí como profesor", especifica una de las entrevistadas, entre otras condiciones (mujer, 14 años dentro, 1 año fuera). Otro dice: "Que tenga amplias facultades para tomar decisiones con autonomía" (hombre, 10 años dentro, 18 años fuera). Otro entrevistado recalcó el liderazgo autoritario del director del establecimiento donde trabajó: "El sistema lo encontré, por decirlo vulgarmente, muy cuadrado. Yo siempre comparaba que el colegio era como un fundo, el patrón de fundo era el director y los demás eran los peones, así es la realidad del profesor, al menos lo que yo pude ver. Entonces la autoridad que tiene el profesor está muy supeditada a lo que dice el director, siendo que es un profesional igual" (hombre, 3 años dentro, 1 año fuera). Las decisiones que los docentes quieren poder tomar no se restringen solo a qué cosas hacer o 
no hacer en clases, sino también a las áreas o niveles en los cuales desempeñarse, según los intereses y la preparación profesional de cada uno: "Sí [me gustaría volver a hacer clases], con la experiencia que tengo hoy [...] De quinto a octavo, segundo ciclo [...] Especialmente séptimo y octavo, orientación [e] inglés" (mujer, 15 años dentro, 12 años fuera).

\subsubsection{Menor carga de trabajo}

Esta categoría recoge las demandas referidas a una cantidad de trabajo apropiada a las horas por las que se está contratado: "Para empezar, tendría que tener tiempo dentro del horario para planificar y para hacer todo, sin llevarme nada a la casa. Porque eso es lo que [me] pasa ahora, ese fue el cambio abismante que tuve cuando dejé de hacer clases: ahora llego a mi casa y no me dedico a seguir trabajando. Tengo vida" (mujer, 7 años dentro, 1 año fuera). En la misma línea, otro participante señala: "Yo salgo de mi trabajo actual y es mi tiempo. O sea, yo no tengo que llegar a trabajar a la casa. Los fines de semana, dispongo de ese tiempo. Y creo que eso tiene que ver con un bienestar personal que también favorece un buen desempeño laboral: si estamos bien como personas, vamos a poder desenvolvernos bien como docentes" (hombre, 1 año dentro, 3 años fuera).

\section{DISCUSIÓN}

Tres de los siete grupos de razones para dejar el aula que hemos identificado en este estudio concuerdan con los hallazgos que han sido reportados tanto en la literatura internacional como en los estudios previos realizados en Chile: el tema de las remuneraciones, la insatisfacción laboral y la desprofesionalización. Se trata, pues, de tres áreas centrales a la hora de pensar en mecanismos para revertir la preocupante tasa de abandono docente que registra nuestro sistema escolar. A la inversa, las dificultades asociadas al mal comportamiento y a la diversidad de los estudiantes, aunque mencionadas con cierta frecuencia en estudios realizados en otros países, no parecen tener tanto peso en la decisión de dejar la enseñanza en Chile.

Un cuarto ámbito bastante central que no aparece mayormente en la investigación internacional, pero sí en el estudio de Ávalos y Valenzuela (2016) y en el nuestro, tiene que ver con las dificultades que tienen en Chile los profesores para seguir estudios de perfeccionamiento sin tener que abandonar el aula. Esta situación resulta especialmente negativa si se considera que la mejora de cualquier sistema educativo pasa en buena medida por la retención de docentes calificados (OECD, 2005). Después de todo, la formación inicial es apenas punto de partida en el proceso de convertirse en profesor, el cual requiere de aprendizajes que solo pueden ocurrir durante el ejercicio mismo de la profesión (FeimanNemser, 1983; Ganser, 2002; Ingersoll \& Strong, 2011).

Por otra parte, dos de las categorías aquí identificadas no suelen ser referidas en otros estudios (ni nacionales ni internacionales): el abandono debido a intereses distintos de la enseñanza en la escuela y el abandono debido a que se asume un cargo de gestión. Que lo primero sea lo más reportado por los participantes nos parece significativo, pero queda para futuras investigaciones determinar a qué se debe. Quizá se trate de un fenómeno más o menos normal en todas las profesiones; alternativamente, podría deberse a un déficit vocacional entre quienes ingresan a la carrera docente (como el caso de la entrevistada que dice que entró a pedagogía porque no pudo ser abogado) o, también, a que las malas 
condiciones laborales (sobrecarga de trabajo, desprofesionalización, bajas remuneraciones, mala gestión) estén llevando a los profesores a considerar otras posibilidades laborales que, en mejores circunstancias, no habrían considerado. Algo similar podría preguntarse respecto de quienes dejan la docencia para asumir un cargo de gestión: ¿es esta elección un modo de incrementar un sueldo que, de otro modo, sería difícil incrementar? ¿Es, tal vez, una forma de superar ese "techo bajo" que algunos han sentido al desempeñarse como profesores?

La información recogida en este estudio no permite dar respuesta a estas preguntas, pero cualquier intento por contestarlas debiese partir por distinguir los tres tipos de abandono especificados en la introducción. Los estudios de abandono docente, por lo general, no hacen distinciones en esta línea y tienden a centrarse exclusivamente en casos del tipo 3. Sin embargo, dado que en los casos de tipo 1 y 2 los involucrados no abandonan el terreno educativo, es posible que en esos casos haya una base vocacional más poderosa que en los casos de tipo 3 y, por lo mismo, aun si no hay un retorno al aula, puede haber disposición a trabajar por mejorar nuestro sistema escolar.

Dicho de manera más general, es perfectamente posible - y razonable- que las motivaciones subyacentes a cada uno de los tres tipos de abandono (y las medidas para prevenirlo) sean bien diferentes. Dejaremos el testeo apropiado de esta hipótesis a investigaciones futuras, aunque el presente estudio nos da algunas pistas. Por ejemplo, de los cinco relatos que aluden a que las acciones desde fuera del aula pueden tener mayor impacto que desde dentro, cuatro de ellos se originan en casos de abandono de tipo 2. Esto podría sugerir que el deseo de impactar a más personas, o a niveles más globales, es parte del sustrato motivacional propio o típico del abandono de tipo 2. Asimismo, pese a que, por definición, todos los casos de abandono de tipo 1 involucran haber asumido un cargo de gestión, de ningún modo es necesario que esa sea una razón — mucho menos la única razón - por la cual se toma la determinación de salir del aula. Esto fue, sin embargo, lo que reportaron de hecho varios de los participantes, incluso aquella entrevistada a la que, según su relato, le encantaba hacer clases. Creemos, por consiguiente, que una exploración más profunda de las razones para asumir un cargo de gestión en la escuela podría revelar un sustrato motivacional frecuente en los casos de abandono de tipo 1.

Respecto del factor remuneraciones, el hecho de que siempre sea mencionado junto a otros aspectos sugiere, a nuestro juicio, que al menos en muchos casos el dinero no es por sí solo un aspecto determinante a la hora de tomar la decisión de dejar el aula. Por otro lado, no hay que perder de vista que el incremento en las remuneraciones es, en efecto, la condición que más se repite entre los entrevistados cuando se les pregunta bajo qué condiciones volverían al aula. Esto nos hace pensar que aun cuando el aspecto económico no sea el que explique mejor el abandono docente, es crucial para atraer profesores al sistema escolar, en especial a aquellos que se han alejado.

La motivación para abandonar la profesión que más peso obtuvo en el estudio de Ávalos y Valenzuela (2016) fue la insatisfacción de los docentes con las oportunidades de desarrollo profesional. Los hallazgos de nuestro estudio permiten describir con mayor detalle en qué puede consistir esta insatisfacción: la sensación de tener el "techo bajo", ligada a una gestión que no logra dar los apoyos necesarios y que incluso puede volverse autoritaria en un contexto que ofrece escasas o nulas facilidades para seguir estudios de perfeccionamiento. Si a esto le sumamos la rigidez de un formato escolar que casi no da cabida a la innovación y al desarrollo de la creatividad, y una sobrecarga de trabajo que no deja un solo momento para la reflexión sobre la práctica, la posibilidad de desempeñarse y 
desarrollarse como profesional de la enseñanza queda radicalmente mutilada. Ha llegado el momento de preguntarnos con la mayor seriedad qué podemos hacer para que nuestras escuelas dejen de ser tan hostiles y aburridas, no ya solo para buena parte de los alumnos, sino también para un grupo preocupantemente alto de profesores.

La recientemente promulgada Ley de Carrera Docente se hace cargo de varias de las razones para dejar el aula aquí consignadas, en particular aquellas relacionadas con las remuneraciones, la sobrecarga laboral (o al menos el exceso de horas dentro del aula) y las oportunidades de perfeccionamiento. Asimismo, las tutorías para acompañar a los profesores neófitos en su proceso de inserción laboral podrían ayudar a disminuir su sensación de insatisfacción laboral. Sin embargo, ni la desprofesionalización ni la mala gestión se ven mayormente alteradas por estas medidas. Tampoco el evaluacionismo frenético de nuestro sistema, que no colabora en nada para que los docentes se sientan a gusto: "El profesor actual vive estresado. Se le generan muchas instancias de medición, de evaluación. Pero se le capacita poco, creo" (hombre, 1 año dentro, 3 años fuera). En esta línea, Gaete, Gómez y Bascopé (2016) han apuntado recientemente que "no tiene sentido seguir generando instancias de evaluación a futuros profesores o a docentes en servicio, sobre todo si no se hacen mejoras concretas a los programas de formación" (p. 13). Los termómetros no sirven para quitar la fiebre: por más evaluaciones que inventemos, no habrá mejoras en el desempeño docente si no se permite a nuestros profesores participar en procesos formativos de calidad sin tener que dejar la docencia.

Asimismo, a los males ya bien sabidos de una rendición de cuentas tan demandante como aquella que opera en el sistema escolar chileno (véase, entre muchos otros, Assael et al, 2011; Biesta, 2015; Biscarra, Giaconi \& Assael, 2015; Contreras \& Corbalán, 2010; Cornejo et al, 2015; Darling-Hammond, 2004; Falabella, 2014; Gaete, 2015; MacBeath, 2012) debe agregarse el efecto que este esquema está teniendo sobre la permanencia de los profesores en dicho sistema. Hace unos años, Assael et al. (2011) plantearon que "los mecanismos de presión, control y rendición de cuentas han afectado seriamente la autonomía profesional, provocando desmoralización en el ejercicio docente, así como elevados niveles de angustia y stress [...]" (p. 317). Si esto es así, los mencionados mecanismos estarían fomentando el abandono docente desde al menos tres frentes: la sobrecarga de trabajo (por el monto de trabajo administrativo que demandan de los profesores), la desprofesionalización (por el ataque a la autonomía) y, por cierto, la insatisfacción laboral (angustia, estrés, etc.). Pero a esto debe agregarse que, en Chile, buena parte de la rendición de cuentas funciona anclada a los resultados SIMCE, lo que contribuye al malestar de aquellos profesores que, como varios de los participantes de este estudio, se han desencantado de la enseñanza orientada a dichos resultados. Por lo demás, la ciudadanía toda tiende a preferir procesos educativos más integrales (véase Gaete \& Ayala, 2015). En consecuencia, nos parece imperioso modificar los mecanismos mediante los cuales se lleva a cabo en Chile la rendición de cuentas, la evaluación docente y la medición de la calidad de la educación. La retención de nuestros docentes depende en buena medida de ello.

\section{REFERENCIAS BIBLIOGRÁFICAS}

Ávalos, B. (2013). Lo que nos enseña la investigación internacional sobre la inserción laboral de los docentes. Santiago: CIAE, Universidad de Chile. 
Ávalos, B., \& Valenzuela, J. P. (2016). Education for all and attrition/retention of new teachers: A trajectory study in Chile. International Journal of Educational Development, 49, 279-290.

Assael, J., Cornejo, R., González, J., Redondo, J., Sánchez, R., \& Sobarzo, M. (2011). La empresa educativa chilena. Educação y Sociedade, 32(115), 305-322.

Barmby, P. (2006). Improving teacher recruitment and retention: the importance of workload and pupil behaviour. Educational research, 48(3), 247-265.

Biesta, G. (2015). What is education for? On good education, teacher judgement, and educational professionalism. European Journal of Education, 50(1), 75-87.

Billingsley, B. S. (1993). Teacher retention and attrition in special and general education: a critical review of the literature. Journal of Special Education, 27, 137-174.

Biscarra, C., Giaconi, C., \& Assael, J. (2015). El docente en la legislación educacional chilena. Psicoperspectivas: Individuo y Sociedad, 14(3), 80-92.

Centro de Políticas Públicas. (2012). Propuestas para una carrera docente. Santiago: Centro de Políticas Públicas UC, Elige Educar.

Contreras, P., \& Corbalán, F. (2010). ¿Qué podemos esperar de la Ley de Subvención Escolar Preferencial? Docencia, 41, 4-16.

Cornejo, R., Albornoz, N., Castañeda, L., Palacios, D., Etcheberrigaray, G. Fernández, R., . . Lagos, J. I. (2015). Prescripciones del trabajo docente en el nuevo marco regulatorio de políticas educativas en Chile 1990-2010. Psicoperspectivas, 14(2), 72-83.

Correa, E., Cividini, M., Fuentealba, R., \& Boerr, I. (Eds.). (2013). Formación e inserción profesional: Desafíos y pistas de facilitación para la profesionalización docente. Santiago: Organización de Estados Iberoamericanos para la Educación de la Ciencia y la Cultura.

Darling-Hammond, L. (2003). Keeping good teachers: Why it matters, what leaders can do? Educational Leadership, 60(8), 7-13.

Darling-Hammond, L. (2004). Standards, Accountability, and School Reform. Teachers College Record, 106(6), 1047-1085.

Falabella, A. (2014). The Performing School: The Effects of Market and Accountability Policies. Education Policy Analysis Archives, 22(70). doi:10.14507/epaa.v22n70.2014

Feiman-Nemser, S. (1983). Learning to Teach. In L. Shulman \& G. Sykes (Eds.), Handbook of teaching and policy (pp. 150-170). New York: Longman.

Gaete, A., \& Ayala, C. (2015). Enseñanza básica en Chile: las escuelas que queremos. Calidad en la educación, 42, 17-59.

Gaete, A., Gómez, V., \& Bascopé, M. (2016). ¿Qué le piden los profesores a la formación inicial docente en Chile? Temas de la Agenda Pública, 11(86), 1-18.

Gaete, M. (2015). Formar profesores de secundaria en Chile. ¿Nuevas reformas, viejos problemas? Revista Iberoamericana de Producción Académica y Gestión Educativa, 2, 1-16.

Ganser, T. (2002). The new teacher mentors: Four trends that are changing the look of mentoring programs for new teachers. American School Board Journal, 189(12), 25-27.

Grossman, P., \& Thompson, C. (2004). District policy and beginning teachers: A lens on teacher learning. Education Evaluation and Policy Analysis, 26(4), 281-301.

Hancock, C. B., \& Scherff, L. (2010). Who will stay and who will leave? Predicting secondary English teacher attrition risk. Journal of Teacher Education, 61(4), 328-338.

Hanushek, E., Kain, J., \& Rivkin, S. (2004). Why public schools lose teachers. Journal of Human Resources, 39(2), 326-254.

Ingersoll, R. (2002). The teacher shortage: A case of wrong diagnosis and wrong prescription. NASSP Bulletin, 86(631), 16-30.

Ingersoll, R., \& Strong, M. (2011). The impact of induction and mentoring programs for beginning teachers: A critical review of the research. Review of Educational Research, 81(2), 201-233.

Klassen, R., \& Chiu, M. (2010). Effect on teachers' self-efficacy and job satisfaction: teacher gender, years of experience, and job stress. Journal of Educational Psychology, 102(3), 741-756. 
Lortie, D. (2002). Schoolteacher (2nd ed.). Chicago: University of Chicago Press.

Luekens, M., Lyter, D., \& Fox, E. (2004). Teacher Attrition and Mobility: Results from the Teacher Follow-up Survey, 2000-01, NCES 2004-301. National Center for Education Statistics, U.S. Department of Education, Washington, D.C.

MacBeath, J. (2012). Future of teaching profession. Cambridge: Educational International Research Institute and University of Cambridge.

Manuel, J. (2003). Have we mistaken the symptom for the problem? Early career teacher retention and attrition. Curriculum \& Leadership Journal, 1(24), 6-8.

McKenzie, P., Santiago, P., Sliwka, P., \& Hiroyuki, H. (2005). Teachers matter: Attracting, developing and retaining effective teachers. Paris: OCDE.

Mizala, A., González, P., Romaguera, P., \& Guzmán, A. (2000). Los maestros en Chile: carreras e incentivos. Inter-American Development Bank. Recuperado de https://publications.iadb.org/ bitstream/handle/11319/6125/Los\%20maestros\%20en\%20Chile\%3a\%20Carreras\%20e\%20 incentivos.pdf?sequence $=1$

OECD. (2001). Schooling for Tomorrow: What Schools for the Future? Paris: OECD.

OECD. (2005). Attracting, Developing, and Retaining Effective Teachers. Paris: OECD.

PricewaterhouseCoopers. (2001). Teacher Workload Study. A Report of a Review commissioned by the DfES. London: PricewaterhouseCoopers.

Skaalvik, E. M., \& Skaalvik, S. (2011). Teacher job satisfaction and motivation to leave the teaching profession: Relations with school context, feeling of belonging, and emotional exhaustion. Teaching and Teacher Education, 27, 1029-1038.

Struyven, K., \& Vanthournout, G. (2014). Teacher's exit decisions: An investigation into the reason why newly qualified teachers fail to enter the teaching profession or why those who do enter do not continue teaching. Teaching and teacher education, 43, 37-45.

Taylor, J., \& Dale, I. (1971). A survey of teachers in their first year of service. Bristol: University of Bristol.

Valenzuela, J., \& Sevilla, A. (2013). La movilidad de los nuevos profesores chilenos en la década del 2000: un sistema escolar viviendo en peligro. Recuperado de http://www.ciae.uchile.cl/ download.php?file=2015-docentes/Movilidad_Valenzuela\%20y\%20Sevilla.pdf

Veenman, S. (1984). Perceived problems of beginning teachers. Review of Educational Research, 54(2), 143-178.

Zehm, S. J. (1999). Deciding to teach: Implications of a self-development perspective. En R. P. Lipka \& T. M. Brinthaupt (Eds.), The Role of Self in Teacher Development (pp. 36-52). New York, NY: SUNY Press. 